\title{
Enlargement of bandgap in 1-D photonic crystal heterostructures
}

\author{
J P Pandey \& Umesh K Pandey \\ M L K P G College, Balrampur (UP)-271201
}

\begin{abstract}
Photonic crystal heterostructures form a natural extension to the analogy between semiconductors and photonic crystals-an analogy which substantially motivated research in photonic band-gap materials. Semiconductor heterostructures are made by combining at least two materials that have distinct band structures. In this paper, a heterostructure is proposed which is a combination of a Fibonacci quasi-periodic structure and a periodic structure. The combined structure has a large omnidirectional reflection range. The condition is that the omnidirectional reflection range of the two sub structures should be adjacent to each other.
\end{abstract}

\section{Introduction}

Photonic band gap materials or photonic crystals (PCs) with large band gap are highly desired in some applications as mirrors, substrates for antennas and waveguides [1,2]. This can be achieved if a PC has a large PBG along the incident direction. This enlargement can be done in different ways: (i) by stacking together two or more different multilayers, i.e. by using photonic heterostructures, $[3,4]$ (ii) by increasing the refractive index contrast [5]; (iii) by using the chirped structure, where the thickness of the period is increased continuously instead of being constant [6]; (iv) by using the disordered structures [7]; (v) by introducing defect in periodic structures [8].

The heterostructure consists of two one dimensional periodic photonic structures with different photonic stop bands. Multiple heterostructures are formed if several 1-D PCs with different layer thicknesses are combined. We found that the band gap of the heterostructures was extended obviously. Heterostructure engineering is now widely practiced, producing the most efficient semiconductor lasers [9], highest-speed transistors [10], and novel quantum electronic devices [11]. If the nontransmission frequency range (PBG along the incident direction) of the constituents has a proper lineup, this can be essentially enlarged as desired by using different PCs to form photonic multiple heterostructures. The directional PBGs for different PCs overlap each other at any incident angle. This kind of lineup of PBGs is crucial for the enlargement of the omnidirectional total reflection by using two or more PCs to form photonic heterostructures. The forbidden transmission frequency range will be from the lowest edge of the nontransmission ranges to the highest upper edge of the nontransmission ranges of the constituent PCs. As a result, the frequency range of total reflection in some sense is enlarged for all incident angles. The idea to use photonic multiple heterostructures may provide a simple and effective way to solve the problem of enlarging the nontransmission frequency region, which may have potential applications $[7,8,12]$.

Zhang et al. [12] has demonstrated that it is possible to enlarge the total reflection frequency range by combining two or more PCs (photonic heterostructures) for 1D and 2D PCs in the case of normal incidence. Li Wang et al. [13] have studied the photonic transmission of the heterostructures with defects and shown that the structure can be used for efficient multiple-channeled optical filtering with enlarged nontransmission frequency ranges. Dong et al. [14] have formed a heterostructure by combining two Fibonacci quasi-periodic structures and a periodic structure and a broad omnidirectional reflection band is obtained. The enlargement of omnidirectional total reflection frequency range of a multilayered dielectric heterostructures is theoretically investigated [15-18]. Three structures of Na3AlF6/Ge multilayer have been studied [15]. The thickness of the two layers of the first and second structure is differing from each other and the third photonic structure is the combination of first and second structures. It is found that the proposed structure has very wide range of omnidirectional reflections bands for both polarizations. Vipin Kumar et al. [16] proposed a simple design of one-dimensional omni-directional reflector based on photonic crystal heterostructures The proposed structure consists of a periodic array of alternate layers of $\mathrm{SiO}_{2}$ and $\mathrm{Te}$ and it has three stacks of periodic structures having five layers each. The lattice period of successive stack is increased by a certain multiple (say gradual constant, $\delta$ ) of the lattice period of the just preceding stack. It is found that such a structure has wider reflection bands in comparison to a conventional dielectric PC structure. Shiqi Wang et al. [19] proposed a new approach to create OBGs by ternary photonic heterostructures (TPHs). It may provide a powerful technique for designing the structures being able to produce OBGs by use of usual materials, low cost materials and materials with low refractive indices etc.

Here, the photonic band gap of the heterostructure formed by the combination of Fibonacci quasiperiodic and periodic 1-D photonic crystal is theoretically investigated. 


\section{Mathematical Formulation}

Fibonacci sequences are multilayer structures consisting of two different materials. Two layers are labeled as $\mathrm{H}$ and $\mathrm{L}$ where $\mathrm{H}$ denotes the material with higher refractive index and $\mathrm{L}$ denotes the material with lower refractive index. A one dimensional quasi periodic Fibonacci sequence is generated by the following recursive relation,

$$
\mathrm{S}_{\mathrm{j}+1}=\left\{\mathrm{S}_{\mathrm{j}-1}, \mathrm{~S}_{\mathrm{j}}\right\}, \quad \mathrm{j} \geq 1
$$

where $S_{j}$ is the $j^{\text {th }}$ generation of the Fibonacci structure. Here $S_{0}=H$ and $S_{1}=L$. In this sequence we have, $S_{0}=H, S_{1}=L, S_{2}=H L, S_{3}=$ LHL, $S_{4}=$ HLLHL etc.

Using Maxwell's boundary conditions that the tangential components of electric and magnetic fields must be continuous at the interface between arbitrary two layers and simplifying, the characteristic matrix for TE (s) and TM (p) waves have the form [20,21]:

$$
\mathrm{T}=\left[\begin{array}{cc}
\cos \beta & -\frac{\mathrm{i}}{\mathrm{q}} \sin \beta \\
-\mathrm{iq} \sin \beta & \cos \mathrm{q}
\end{array}\right]
$$

where for TE wave $\mathrm{q}=\mathrm{n} \cos \theta$, and for TM wave $\mathrm{q}=\frac{\cos \theta}{\mathrm{n}}, \beta=2 \pi \mathrm{nd} \cos \theta / \lambda, \theta$ is the angle of incident. Thus, the transfer matrices are $T_{0}=T_{H}, T_{1}=T_{L}, T_{2}=T_{H} T_{L}, T_{3}=T_{L} T_{H} T_{L}$ and $T_{4}=T_{H} T_{L} T_{L} T_{H} T_{L}$ for $S_{0}, S_{1}, S_{2}$, $\mathrm{S}_{3}$ and $\mathrm{S}_{4}$ respectively. Considering the structure consisting of $\mathrm{N}$ layers of quasi-periodic Fibonacci structure $\mathrm{S}_{j}$ and $\mathrm{N}^{\prime}$ layers of periodic structure $\mathrm{S}_{2}$, then the total characteristic matrix is given by,

$$
\mathrm{T}=\left(\mathrm{T}_{\mathrm{j}}\right)^{\mathrm{N}}\left(\mathrm{T}_{2}\right)^{\mathrm{N}^{\prime}}=\left[\begin{array}{ll}
\mathrm{T}_{11} & \mathrm{~T}_{12} \\
\mathrm{~T}_{21} & \mathrm{~T}_{22}
\end{array}\right]
$$

The reflection coefficient is given by,

$$
r=\frac{\left(\mathrm{T}_{11}+\mathrm{q}_{\mathrm{t}} \mathrm{T}_{12}\right) \mathrm{q}_{\mathrm{i}}-\left(\mathrm{T}_{21}+\mathrm{q}_{\mathrm{t}} \mathrm{T}_{22}\right)}{\left(\mathrm{T}_{11}+\mathrm{q}_{\mathrm{t}} \mathrm{T}_{12}\right) \mathrm{q}_{\mathrm{i}}+\left(\mathrm{T}_{21}+\mathrm{q}_{\mathrm{t}} \mathrm{T}_{22}\right)}
$$

where $\mathrm{q}_{\mathrm{i}, \mathrm{t}}=\mathrm{n}_{\mathrm{i}, \mathrm{t}} \cos \theta_{\mathrm{i}, \mathrm{t}}$ for TE wave and $\mathrm{q}_{\mathrm{i}, \mathrm{t}}=\frac{\cos \theta_{\mathrm{i}, \mathrm{t}}}{\mathrm{n}_{\mathrm{i}, \mathrm{t}}}$ for TM wave, where $\mathrm{i}$ and $\mathrm{t}$ represent incident medium and substrate respectively. The reflectivity is given by,

$$
\mathrm{R}=|\mathrm{r}|^{2}
$$

\section{Results and Discussion}

Here, two dielectric materials $\mathrm{MgF}_{2}$ and $\mathrm{Si}$ are taken whose refractive indices are 1.38 and 3.7 respectively at the wavelength of $700 \mathrm{~nm}$. The structure is supposed to be on a substrate of glass with refractive index 1.5. The thicknesses are taken according to the quarter wave arrangement. Fibonacci structure $\mathrm{S}_{4}$ and periodic structure $S_{2}$ each having 10 periods will be investigated. Here, all the regions are assumed to be linear, homogeneous and non-absorbing.

The omnidirectional photonic band gap for both TE and TM polarization is defined by the upper photonic band gap edge at $90^{\circ}$ incident angle and the lower photonic band gap edge at normal incidence. The omnidirectional photonic band gap for TM polarization lies completely within omnidirectional photonic band gap of TE polarization. Therefore, complete omnidirectional photonic band gap is the omnidirectional photonic band gap of TM polarization. The reflectance spectra of two photonic crystals are shown in figure 1 and figure 2 at different angles. Figure 1 shows the reflectance spectra of Fibonacci photonic crystal. Omnidirectional photonic band gap for TE polarization is displayed in the wavelength range from $7436 \mathrm{~A}^{0}-11022 \mathrm{~A}^{0}$. Omnidirectional photonic band gap for TM polarization ranges from $7436 A^{0}-9123 A^{0}$. Therefore, omnidirectional photonic band gap for any polarization of this Fibonacci structure spans from $7436 \mathrm{~A}^{0}-9123$ $\mathrm{A}^{0}$. The reflectance spectra of periodic structure have been shown in figure 2 . From this figure, the omnidirectional photonic band gap for TE polarization has its range from $5412 \mathrm{~A}^{0}-9282 \mathrm{~A}^{0}$. For TM polarization, omnidirectional photonic band gap ranges from $5412 \mathrm{~A}^{0}-7604 \mathrm{~A}^{0}$. Hence, omni PBG for any polarization for this periodic structure is $5412 \mathrm{~A}^{0}-7604 \mathrm{~A}^{0}$. Comparing the reflectance spectra of the two structures, for the same number of layers, 20, the omnidirectional photonic band gap range is large for the periodic structure as compared to quasi-periodic structure. The reflectance spectra of the third photonic structure which is the combination of Fibonacci quasiperiodic and periodic structure (figure 3) show that the 
omnidirectional photonic band gap for TE polarization ranges from $4912 \mathrm{~A}^{0}-11022 \mathrm{~A}^{0}$. For TM polarization, this range is from $4912 \mathrm{~A}^{0}-9123 \mathrm{~A}^{0}$. Therefore, omnidirectional photonic band gap for any polarization has its range from $4912 \mathrm{~A}^{0}-9123 \mathrm{~A}^{0}$ for the combined structure. Comparing the reflectance spectra of the three structures, it can be seen that the combined structure has a large omnidirectional reflection range, larger than the sum of the omnidirectional reflection ranges of Fibonacci quasiperiodic structure and periodic structure. Figures 1, 2 and 3 show the conventional photonic band diagrams for the three considered structures. Figure 4 shows that Fibonacci structure has two high reflection bands. But it is clear from the figure that the band at the lower wavelength side does not exhibit an omnidirectional reflection band but the other band at higher wavelength side has an omnidirectional band gap. The periodic structure has only one high reflection band as shown in figure 5 and this band also represents an omnidirectional band gap. Surprisingly, the two bands for Fibonacci structure are on the both sides of the band of the periodic structure. Photonic band diagram for the combined structure is shown in figure 6. It has only one high reflection band which is very large as compared to Fibonacci and periodic structures. Thus, the two reflection bands formed by the Fibonacci structure are compensated by the reflection band of the periodic structure. So, a large omnidirectional band gap is obtained which encroaches the region of non-omnidirectional high reflection band in the Fibonacci structure.
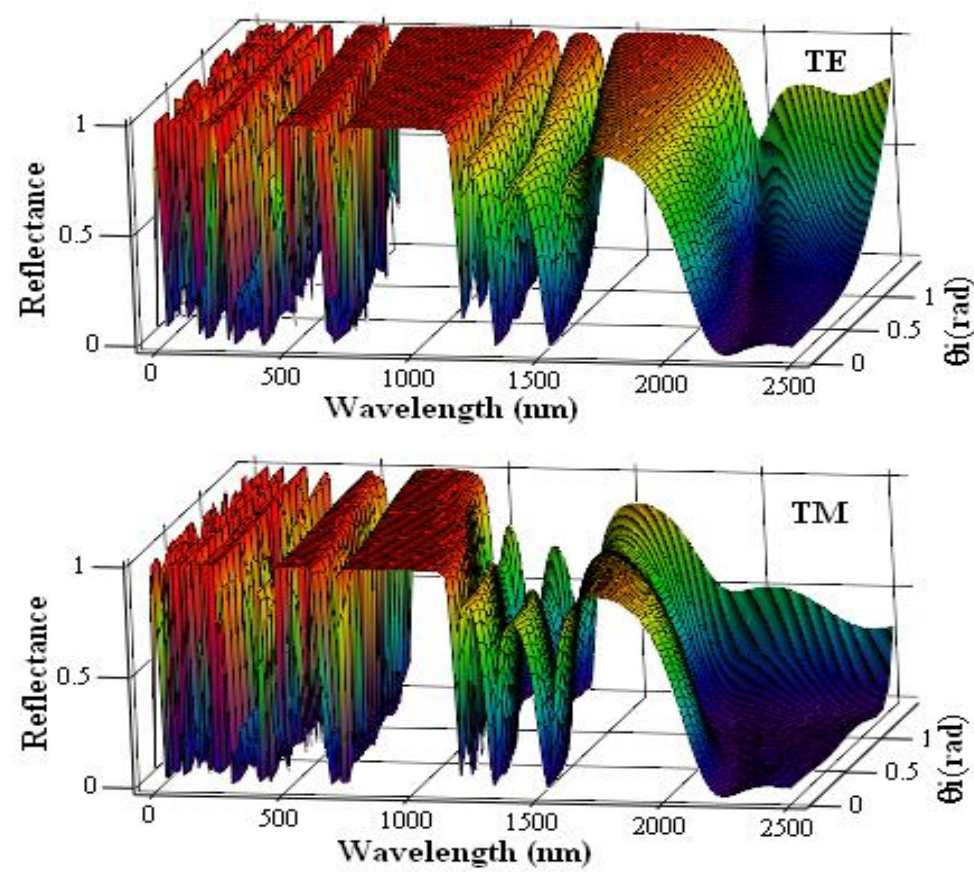

(a)
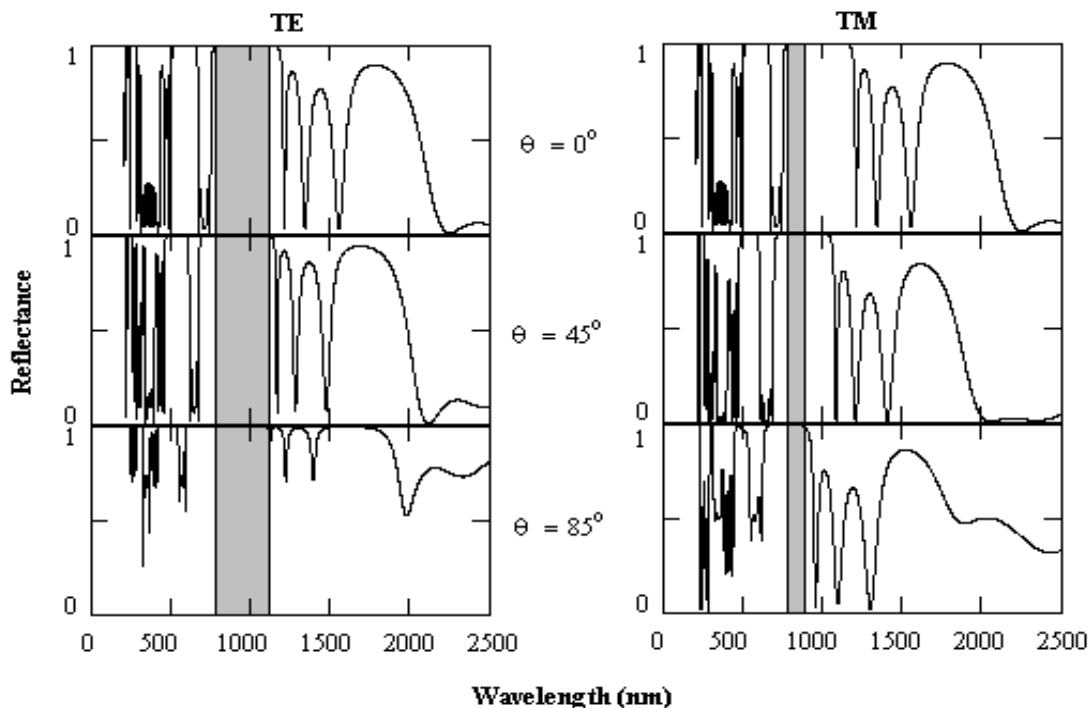

(b)

Fig. 1 Reflectance spectra of Fibonacci quasi-periodic structure for TE and TM polarizations. 

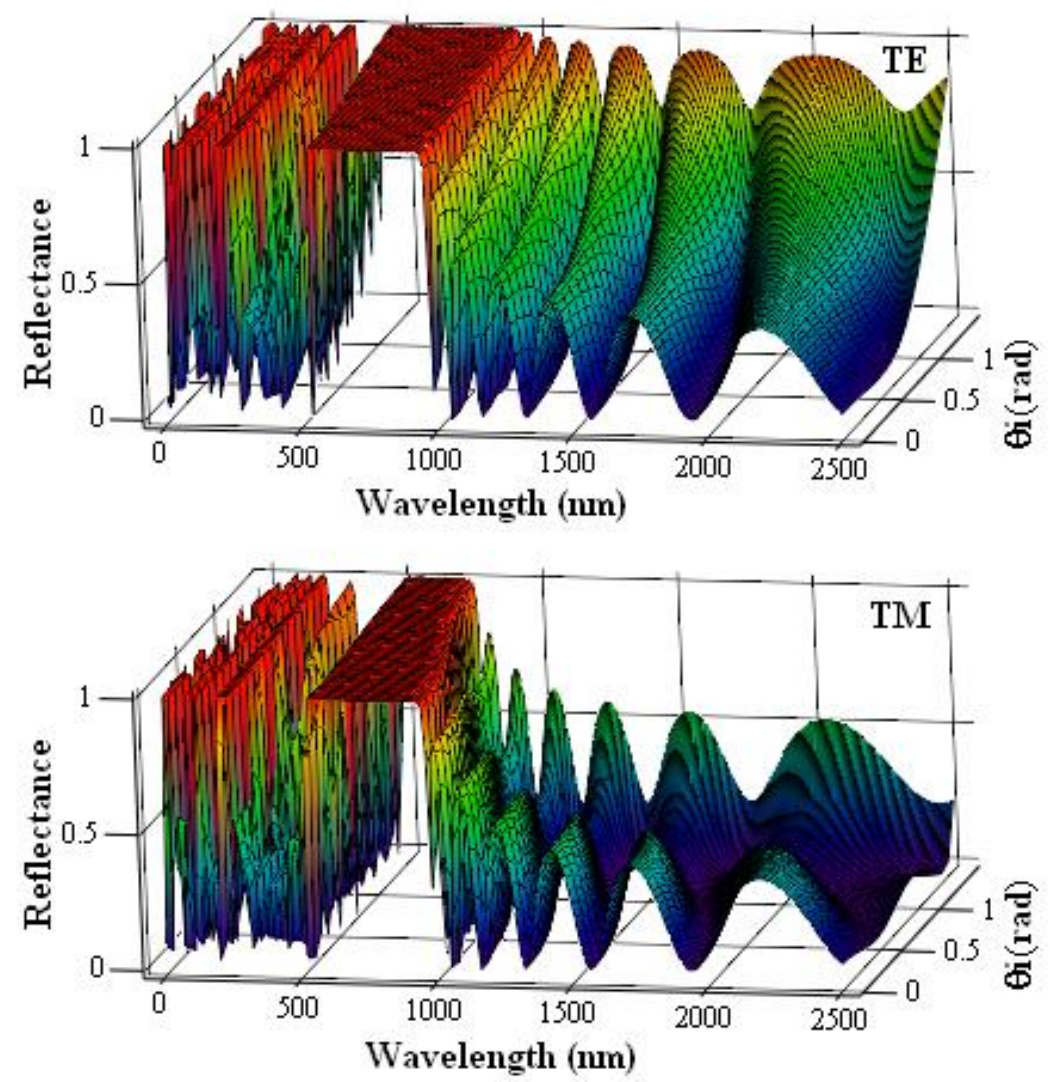

(a)
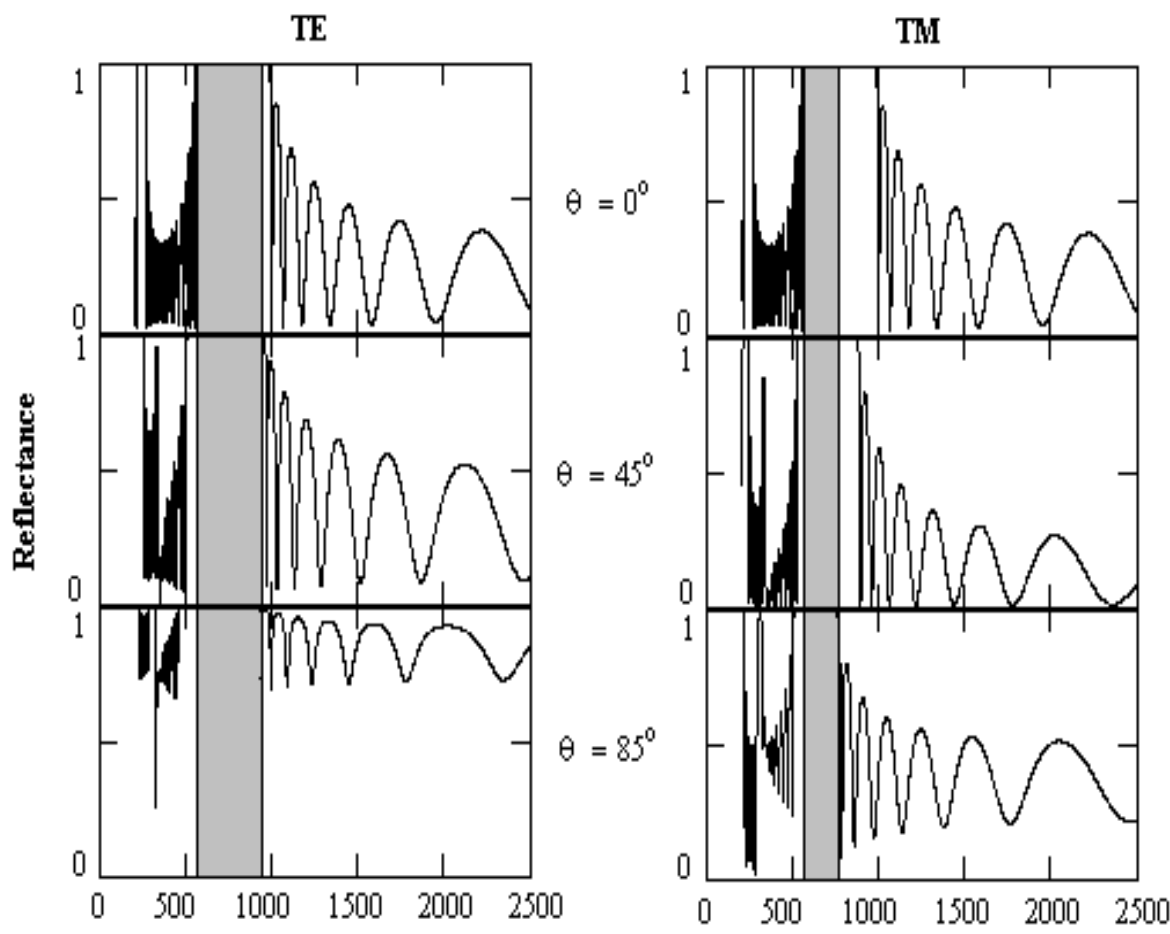

\section{Wavelength (um)}

(b)

Fig. 2 Reflectance spectra of Periodic structure for TE and TM polarizations. 

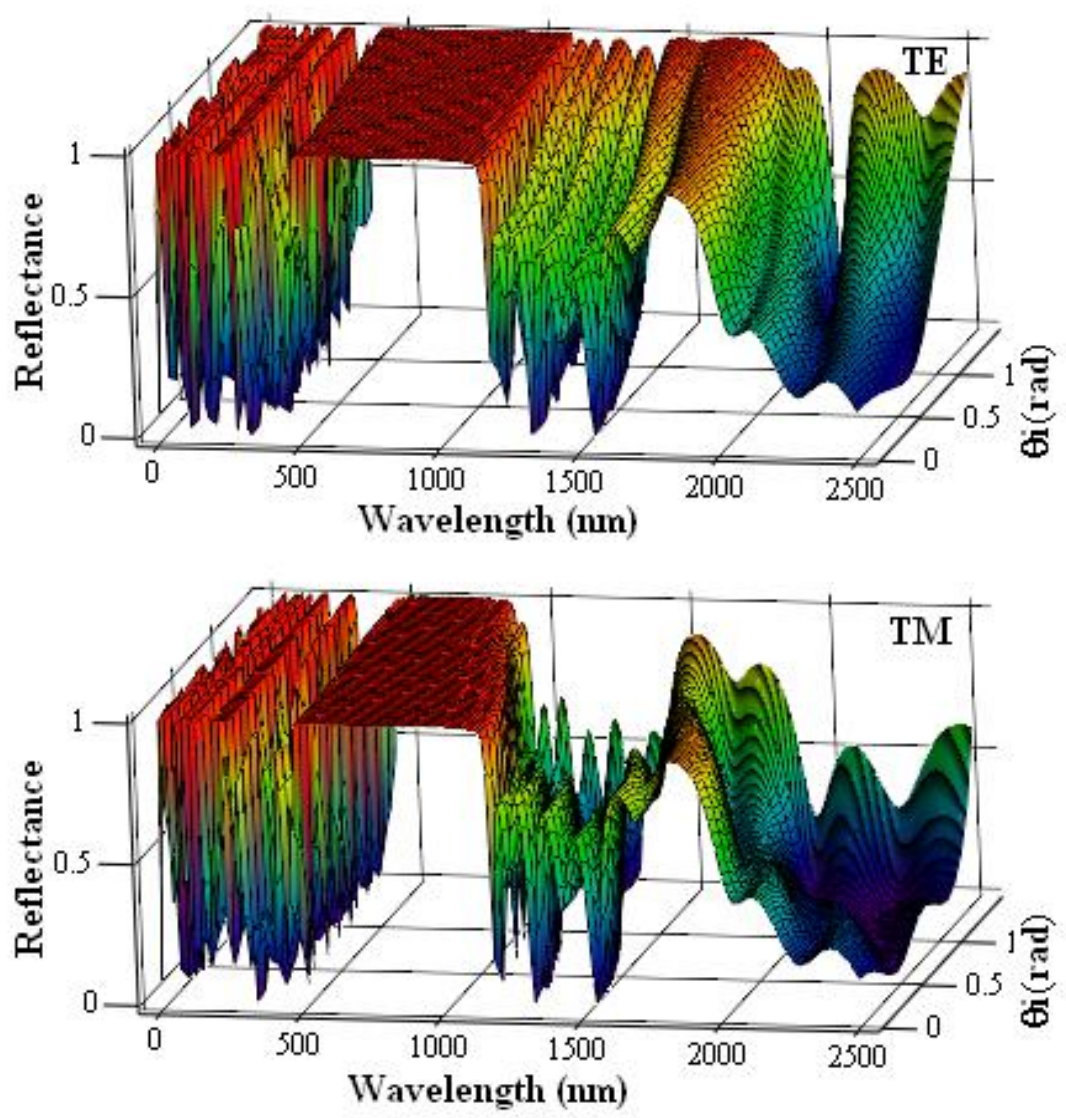

(a)
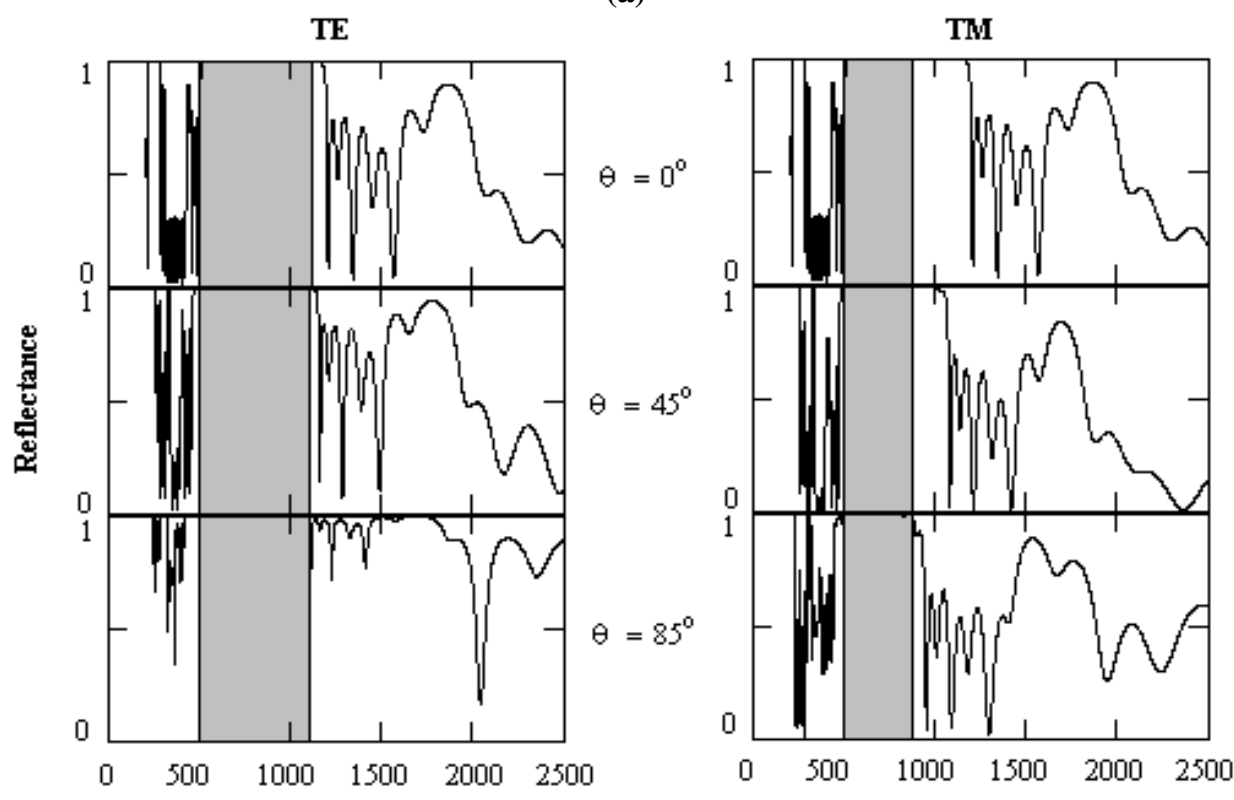

Wavelength (un)

(b)

Fig. 3 Reflectance spectra of combination of Fibonacci quasi-periodic structure and periodic structure for TE and TM polarizations. 


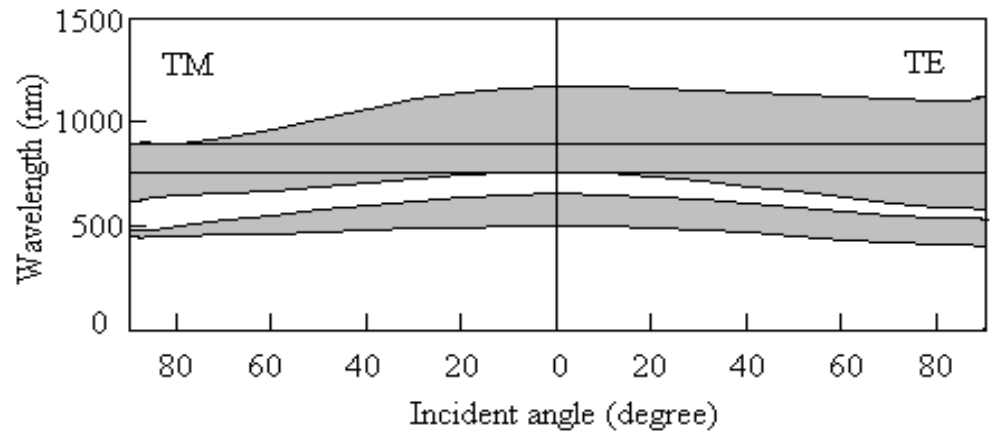

Fig. 4 Photonic band structure of Fibonacci quasi-periodic structure.

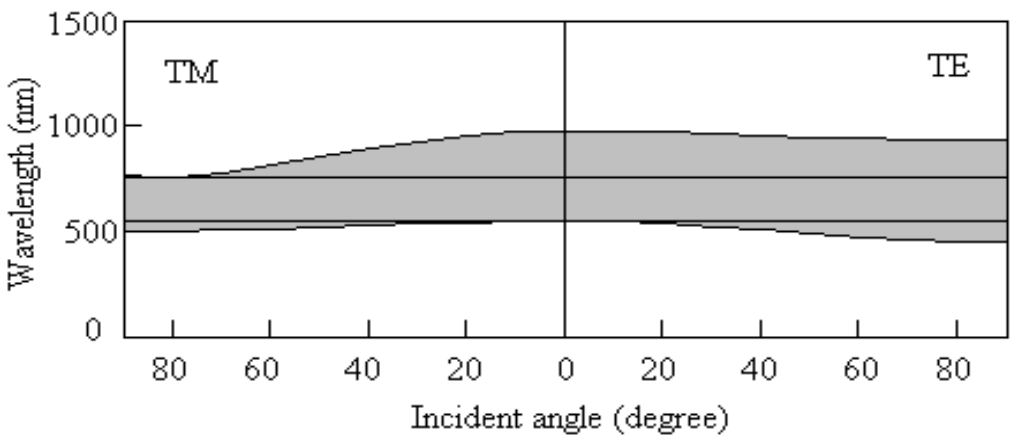

Fig. 5 Photonic band structure of periodic structure.

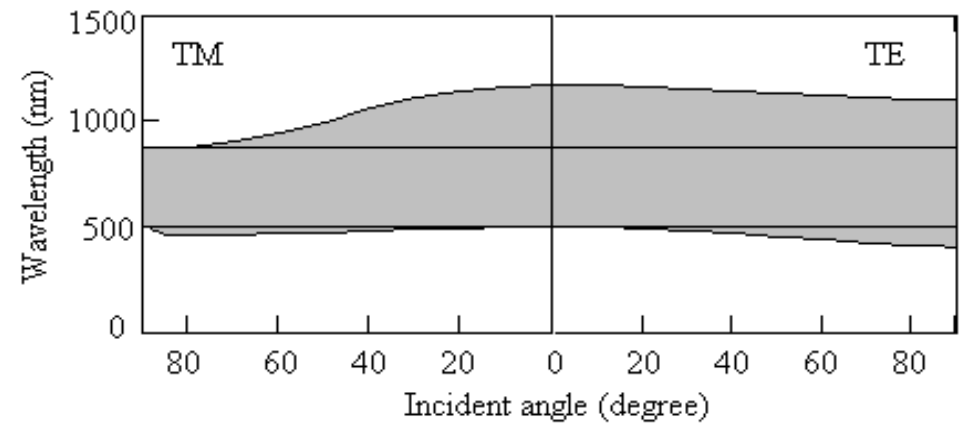

Fig. 6 PBG structure of combination of Fibonacci quasi-periodic structure and periodic structure

\section{References}

[1]. J. S. I, Y. Park and H. Jeon, J. Lightwave Technol. 22, 509, 2004.

[2]. R. Sauleau, P. Coquet, T. Matsui, and J. P. Daniel, IEEE Trans. Antennas Propag. 51, 3171, 2003.

[3]. J. Zi, J. Wan, and C. Zhang, Appl. Phys. Lett. 73, 2084-2086, 1998.

[4]. X. Wang, X. Hu, Y. Li, W. Jia, C. Xu, X. Liu, and J. Zi, Appl. Phys. Lett. 80, 4291, 2002.

[5]. J. Lekner, J. Opt. A, Pure Appl. Opt. 2, 349, 2000.

[6]. E. Yablonovitch, Opt. Lett. 23, 1648, 1998

[7]. J. Xu, H. Fang and Z. Lin, J. Phys. D: Appl. Phys. 34, 445-449, 2001.

[8]. H-Y. Lee and T. Yao, J. Appl. Phys. 93, 819-830, 2003.

[9]. W. T. Tsang, Appl. Phys. Lett. 40, 217-219, 1982.

[10]. W. Hafez and M. Feng, Appl. Phys. Lett. 86, 152101, 2005

[11]. H.Sakaki, Jpn. J. Appl. Phys., Part 2 21, L381-L383, 1982.

[12]. Chun Zhang, Feng Qiao, Jun Wan and Jian Zi, J. Appl. Phys. Vol. 87, No. 6, 15 March 2000.

[13]. Li Wang, Z. Wang, Y. Wu, L. Chen, S. Wang, X. Chen, and Wei Lu, J. Appl. Phys. Vol. 95, No. 2, 424-426, 2004.

[14]. Dong Jian-Wen, Han Peng, and Wang He-Zhou, Chin. Phys. Lett., Vol. 20, No. 11, 1963-1965, 2003.

[15]. R. Srivastava, ShyamPati and S. P. Ojha, Progress In Electromagnetics Research B, Vol. 1, 197-208, 2008.

[16]. Vipin Kumar, Mohd. Anis, Kh. S. Singh and Gulbir Singh, Optik - International Journal for Light and Electron Optics, Volume 122, Issue 24, Pages 2186-2190, December 2011.

[17]. J P Pandey, International Journal of Pure and Applied Physics, Volume 13, Number 2 pp. 167-173, 2017

[18]. J P Pandey, IOSR Journal of Applied Physics (IOSR-JAP), Volume 9, Issue 2, pp 59-63 Ver. I (Mar. - Apr. 2017).

[19]. Shiqi Wang, Xiangbo Yang, Chengyi Timon Liu' Physics Letters A, Volume 378, Issues 18-19, pp 1326-1332, 28 March 2014.

[20]. P. Yeh, Optical Waves in Layered Media, New York: John Wiley \& Sons, 1988.

[21]. M. Born, and E. Wolf, Principles of Optics, Cambridge: Cambridge University Press, 1998. 